\title{
Effect of Distension of the Bladder and of Contraction of Sphincters on Blood Pressure
}

\author{
J. J. G. SZASZ*; H. M. WHYTE, $†$ D.PHII., F.R.A.C.P.
}

\author{
Brit. med. F., 1967, 2, 208-210
}

Distension of the bladder has been shown to cause a rise in blood pressure in anaesthetized dogs (Talaat, 1937) and cats (Watkins, 1938) and in patients with spinal cord injuries (Guttmann and Whitteridge, 1947 ; Cunningham et al., 1953), but whether the association is operative and of any clinical significance in normal circumstances is less clear. Observations by Adams-Ray and Norlén (1951) and by More (quoted by Whitteridge, 1960) showed that vasoconstriction occurred in normal subjects when the bladder was uncomfortably full and the intravesical pressure exceeded $50 \mathrm{~mm}$. $\mathrm{Hg}$. Further indirect evidence of an association comes from observations that blood pressure falls after prostatic obstruction is relieved (O'Conor, 1920), of hypertensive crises in patients with grossly distended bladders, and of " micturition syncope" (Lyle et al., 1961). In all instances it seemed that gross distension and perhaps discomfort were required to produce an effect on blood pressure.

The aim of the present study was to assess the practical significance of the problem by carrying out simple experiments in normal subjects and in catheterized patients.

\section{Methods}

Blood pressure was measured by one observer to the nearest $2 \mathrm{~mm}$. $\mathrm{Hg}$ with one standard sphygmomanometer in accordance with the recommendations of the American Heart Association. Duplicate measurements were made on each occasion.

Oral Fluid Load Test.-The response to an oral fluid load of $1,200 \mathrm{ml}$. of flavoured tap-water drunk in 30 minutes was tested in seven healthy volunteers aged 26 to 71 years, mean 38, and four patients of both sexes aged 41 to 77 years, mean 56 . The patients agreed to participate in this study, and were selected because they were in relatively good general condition but had indwelling catheters as part of their treatment for various urological conditions-urethral stricture, prostatic hypertrophy, or vesical diverticula with ureteric reflux. Procedures were explained to subjects before the test but no emphasis was placed on bladder distension. Tests were begun with an empty bladder and not less than one hour after a meal. Subjects were kept sitting during tests, and no eating, drinking, or smoking was allowed. In the initial control period blood pressure was recorded every 10 minutes till the average of duplicate readings remained relatively constant (within $5 \mathrm{~mm}$. $\mathrm{Hg}$ : control value C). After the subjects began to drink the fluid load readings were made every 15 or 30 minutes (see Table I), and they were encouraged to delay emptying the bladder for as long as possible. Further readings were made when bladder distension became intolerable (value D), five minutes after emptying (value E), and subsequently at intervals of 15 minutes. Observations were also made of pulse rate, changes in pupil size, palmar sweating, colour changes of the face or extremities, irritability and signs or complaints of discomfort, and the volume of

- Medical student.

† Director of Medical Research, Kanematsu Memorial Institute, Sydney Hospital, Sydney, Australia. Now Professor of Clinical Science, John Curtin School of Medical Research, Australian National University, Canberra. urine passed. Bladder pressures were recorded in the four patients with urethral catheters.

Retrograde Filling Test.-Two patients had the oral load first and the retrograde test second, while the order was reversed in the other two. The same general procedure was followed as for the oral fluid load test, but the bladder was filled by introducing $50 \mathrm{ml}$. of normal saline through the catheter every 15 minutes till distension was intolerable. Bladder pressure was measured with a saline-filled manometer.

Other observations were made in normal subjects before and after normal micturition and in relation to voluntary contraction of sphincters, as described under Results. Standard statistical methods were used in analysing results.

\section{Results}

Oral Fluid Load.-The initial control values of blood pressure and the changes observed at different times during the course of the oral fluid load test in 11 subjects are set out in Table I. In every case blood pressure was raised at the height of bladder distension, the increase ranging from 4 to 52 (mean 27) $\mathrm{mm}$. $\mathrm{Hg}$ for systolic pressure and 4 to 43 (mean 22) $\mathrm{mm}$. $\mathrm{Hg}$ for diastolic pressure. Mean values are illustrated in Fig. 1. The rise is highly significant $(P<0.01$ systolic and $P<0.001$ diastolic). The time taken for the bladder to fill varied from 50 to 215 minutes. There was no relation between the rise in blood pressure and the extent of bladder distension as judged by the volume of urine subsequently expelled. The blood pressure fell rapidly after the bladder was emptied, and then remained at about the control value in most instances. Exceptions were Subject No. 6, who was having treatment with barbiturates for hypertension, and Subject No. 4, who was disturbed by information that she was to be given a blood trans-

TABLE I.-Control Systolic and Diastolic Blood Pressures and the Changes (mm. Hg) Observed at Different Times After Beginning Tests with Oral Fluid Loading and with Retrograde Filling of the Bladder

\begin{tabular}{|c|c|c|c|c|c|c|c|c|c|c|c|c|c|c|}
\hline \multirow{3}{*}{$\underset{\text { ject }}{\text { Sub- }}$} & \multirow{2}{*}{\multicolumn{2}{|c|}{$\begin{array}{c}\text { Control } \\
\text { Blood } \\
\text { Pressure }\end{array}$}} & \multicolumn{12}{|c|}{ Changes in B.P. During Tests } \\
\hline & & & $\mathbf{S}$ & $\mathbf{D}$ & $\mathbf{S}$ & D & $\mathbf{S}$ & $\mathbf{D}$ & $\mathbf{S}$ & $\mathbf{D}$ & $\mathbf{S}$ & $\mathrm{D}$ & $\mathrm{S}$ & D \\
\hline & $\mathbf{S}$ & $\mathrm{D}$ & \multicolumn{2}{|c|}{$30 \mathrm{~min}}$. & \multicolumn{2}{|c|}{$60 \mathrm{~min}}$. & \multicolumn{2}{|c|}{ Time D } & \multicolumn{2}{|c|}{ Time $\mathrm{E}$} & \multicolumn{2}{|c|}{$+30 \mathrm{~min}}$. & \multicolumn{2}{|c|}{$+60 \mathrm{~min}$} \\
\hline
\end{tabular}

\begin{tabular}{|c|c|c|c|c|c|c|c|c|c|c|c|c|c|c|}
\hline \multirow{3}{*}{$\begin{array}{r}1 \\
2 \\
3 \\
4 \\
5 \\
6 \\
7 \\
8 \\
9 \\
10 \\
11\end{array}$} & \multicolumn{14}{|c|}{ Oral Fluid Load } \\
\hline & $\begin{array}{l}134 \\
112 \\
105 \\
115 \\
109 \\
155 \\
146 \\
130 \\
125 \\
119 \\
119\end{array}$ & $\begin{array}{l}74 \\
66 \\
66 \\
56 \\
66 \\
77 \\
82 \\
70 \\
90 \\
76 \\
79\end{array}$ & $\begin{array}{r}0 \\
4 \\
1 \\
49 \\
9 \\
11 \\
33 \\
6 \\
4 \\
0 \\
10\end{array}$ & $\begin{array}{r}6 \\
8 \\
7 \\
5 \\
11 \\
27 \\
14 \\
7 \\
4 \\
2 \\
6\end{array}$ & \begin{tabular}{|r}
2 \\
2 \\
8 \\
27 \\
27 \\
6 \\
-10 \\
32 \\
-3 \\
4 \\
3
\end{tabular} & \begin{tabular}{|r}
10 \\
8 \\
7 \\
10 \\
3 \\
8 \\
8 \\
16 \\
-1 \\
7 \\
4
\end{tabular} & $\begin{array}{r}52 \\
38 \\
11 \\
25 \\
45 \\
12 \\
40 \\
38 \\
4 \\
12 \\
22\end{array}$ & \begin{tabular}{|r|}
40 \\
24 \\
24 \\
10 \\
18 \\
43 \\
29 \\
30 \\
4 \\
6 \\
10
\end{tabular} & \begin{tabular}{|r}
8 \\
10 \\
4 \\
15 \\
3 \\
-8 \\
11 \\
6 \\
-2 \\
0 \\
7
\end{tabular} & $\begin{array}{r}16 \\
8 \\
8 \\
5 \\
11 \\
30 \\
17 \\
8 \\
1 \\
4 \\
10\end{array}$ & $\begin{array}{r}-2 \\
2 \\
-3 \\
20 \\
5 \\
-1 \\
12 \\
-8 \\
-6 \\
-6 \\
11\end{array}$ & $\begin{array}{r}6 \\
0 \\
6 \\
8 \\
9 \\
27 \\
9 \\
0 \\
-2 \\
4 \\
10\end{array}$ & $\begin{array}{r}-2 \\
4 \\
-3 \\
20 \\
6 \\
10 \\
8 \\
-7 \\
1 \\
-3 \\
13\end{array}$ & \begin{tabular}{|r}
8 \\
0 \\
5 \\
4 \\
7 \\
42 \\
8 \\
0 \\
5 \\
-1 \\
10
\end{tabular} \\
\hline & & & 15 & & $30 \mathrm{n}$ & in. & $\mathrm{Tim}$ & D & Tim & $\mathrm{E}$ & +3 & in. & & $\min$ \\
\hline
\end{tabular}
\begin{tabular}{r|r|r|r|r|r|r|r|r|r|r|r|r|r|r|}
4 & 132 & 61 & 25 & 2 & -2 & -2 & 25 & 10 & -6 & 1 & -10 & 5 & 13 & -6 \\
5 & 106 & 63 & 1 & 0 & 3 & 0 & 19 & 18 & 1 & 0 & -2 & 2 & -2 & 0 \\
8 & 125 & 79 & 7 & -4 & & & 45 & 31 & 12 & -4 & 2 & -9 & 2 & 1 \\
11 & 126 & 82 & 2 & 4 & 4 & 8 & 20 & 20 & 5 & 7 & 7 & 7 & -2 & 6
\end{tabular}

Time $D$ is at maximum tolerable distension of the bladder and $E$ after emptying. 
fusion after the test. Pulse rate did not vary significantly. Bladder distension caused discomfort in all subjects, pain in two, and was accompanied by irritability in eight, palmar sweating in six, and dilatation of the pupil in three. It was thought that the rise in blood pressure at 30 minutes, immediately after drinking the fluid load, might be related to distension of the stomach and small intestine, but it was only of borderline significance.

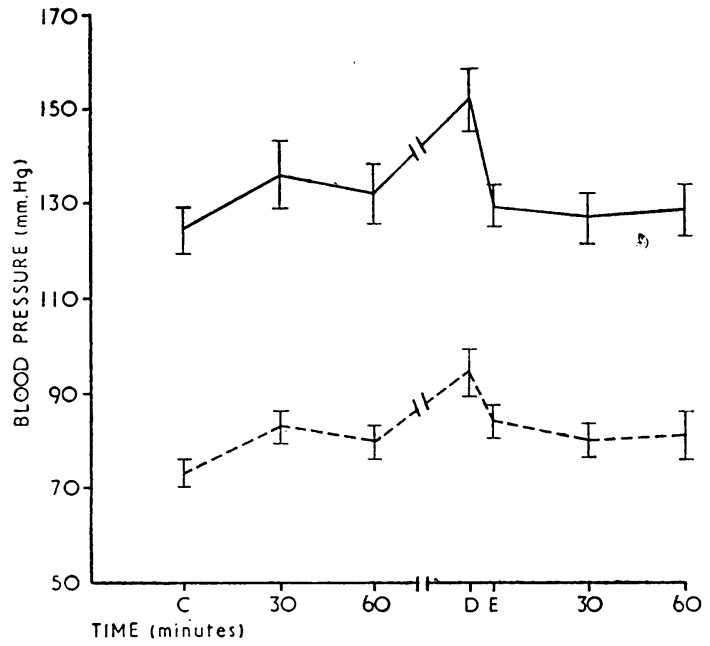

Fig. 1.-Blood pressure after an oral fluid load: mean systolic and diastolic pressures ( $t$ one standard error) for 11 subjects. $C$, control value; $D$, at time of maximum tolerable bladder distension; E, after emptying the bladder.

Retrograde Filling.-A similar pattern of results was produced by retrograde filling of the bladder in four subjects (Table I and Fig. 2). The systolic pressure rose 19 to 45 (mean 27) $\mathrm{mm}$. $\mathrm{Hg}$ and the diastolic pressure 10 to 31 (mean 20) $\mathrm{mm}$. $\mathrm{Hg}$.

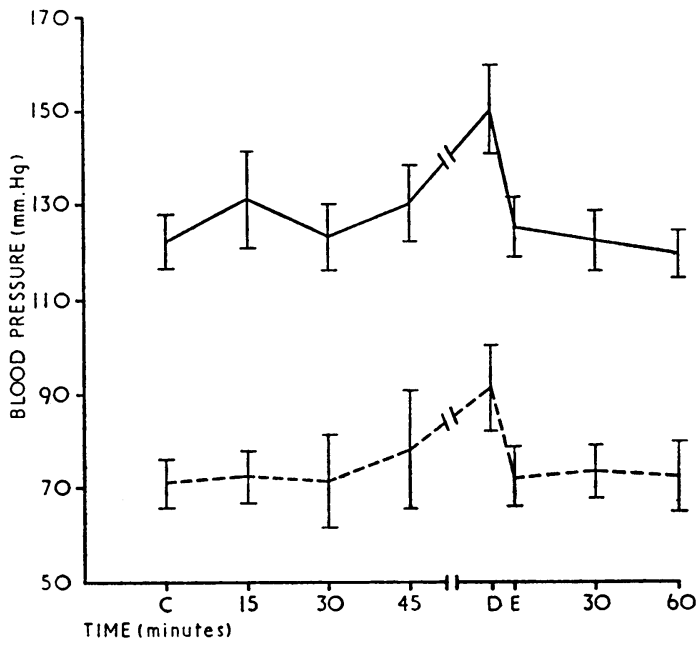

FIG. 2.-Blood pressure with retrograde filling of the bladder: mean values ( $t$ one standard error) for four

Blood Pressure and Bladder Pressure.-Both of these measurements were made during oral and retrograde procedures in four subjects. At the point where emptying the bladder became imperative both blood pressure and bladder pressure rose steeply. Previous to this point being reached no clear relation between blood pressure and bladder pressure was apparent within each individual, but the combined results showed a significant correlation: $\mathrm{r}=0.50, \mathrm{P}<0.02$ (Fig. 3). At the time when subjects first noticed bladder discomfort the average pressure was $20 \mathrm{~cm}$. of water, and their blood pressure was insignificantly higher than the control values.
Normal Micturition.-Duplicate measurements of blood pressure were taken immediately before and after micturition on two successive days in 11 normal subjects. Subjects were asked to follow their habitual practice in deciding when to micturate. In a majority of observations the blood pressure was slightly greater (maximum $6 \mathrm{~mm}$. $\mathrm{Hg}$ ) before micturition, but the analysis of variance showed no significant difference overall. The mean values were $109.2 / 68.0 \mathrm{~mm}$. $\mathrm{Hg}$ before and 106.9/67.2 $\mathrm{mm} . \mathrm{Hg}$ after micturition.

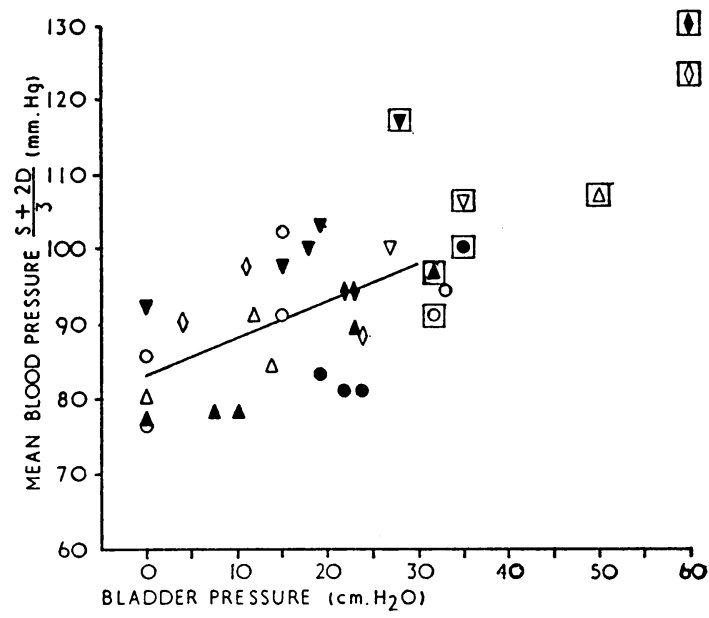

Fig. 3-Mean blood pressure compared with bladder pressure in four subjects tested by both retrograde and orai procedures: excluding values at time $D$ (enclosed in procedures: excluding values at time D (enclosed in squares), $\mathrm{r}=+0.50(\mathrm{P}<$ Observations for the oral test are $5, \triangle ; 8, \diamond ; 11, \nabla ;$ Observations for the oral test are
shown as open symbols and for the retrograde test as closed symbols.

Effect of Voluntary Contraction of Sphincters.-The possibility that the rise in blood pressure associated with filling of the bladder was related to contraction of the sphincter rather than to distension of the bladder was tested in 10 normal subjects. Blood pressure was measured during a control period as described previously and then five times at intervals of three minutes while the subject voluntarily contracted or relaxed his sphincters. Each subject chose his own sequence of behaviour, and disclosed it to the observer only at the end of the test. Altogether there were 28 periods of contraction and 22 of relaxation. In every subject the blood pressure was higher during contraction than during relaxation or the control period. The mean results are shown in Table II. The rise in blood pressure was significant: for systolic pressure, $P<0.001$, and for diastolic pressure, $0.02>\mathrm{P}>0.01$. The pulse rate, measured from E.C.G. recordings made during the tests carried out in four subjects (with 10 periods of contraction and 10 of relaxation), rose significantly $(0.01>\mathrm{P}>0.001)$ from a mean of 67 during periods of relaxation to a mean of 78 during periods when the sphincters were contracted.

TABLE II.-Mean Blood Pressures ( \pm Standard Error, $\mathrm{mm}$. $\mathrm{Hg}$ ) for 10 Subjects During Tests of Voluntary Contraction and Relaxation of Sphincters

\begin{tabular}{|c|c|c|c|}
\hline & \multirow{2}{*}{$\begin{array}{l}\text { Control } \\
\text { Period }\end{array}$} & \multicolumn{2}{|c|}{ Sphincter } \\
\hline & & Contracted & Relaxed \\
\hline $\begin{array}{l}\text { Systolic B.P. } \\
\text { Diastolic B.P. }\end{array}$ & $\begin{array}{r}110 \cdot 8 \pm 3 \cdot 4 \\
67 \cdot 0 \pm 4 \cdot 1\end{array}$ & $\begin{array}{r}128 \cdot 7 \pm 2 \cdot 6 \\
81 \cdot 4 \pm 2 \cdot 0\end{array}$ & $\begin{array}{r}112 \cdot 8 \pm 2 \cdot 5 \\
70 \cdot 6 \pm 2 \cdot 6\end{array}$ \\
\hline
\end{tabular}

\section{Discussion}

It has been clearly established that blood pressure rises when the bladder is distended to the point of discomfort, at which time there is an urgent desire to micturate and strong contraction of the bladder and sphincters. The rise in blood pressure 
is quite pronounced-up to $50 \mathrm{~mm}$. $\mathrm{Hg}$ systolic and $40 \mathrm{~mm}$. $\mathrm{Hg}$ diastolic-and certainly sufficient to be of clinical importance in some patients.

The hypertensive effect is evident when the bladder distension becomes intolerable. There was some suggestion that blood pressure rises gradually as the tension in the bladder rises, even before any sense of discomfort or desire to micturate, but this came only from the pooled observations from the four catheterized patients, and was not substantiated by the blood-pressure measurements made before and after normal micturition. It can be concluded that the average behaviour with respect to retaining one's urine and passing it is not associated with any significant fluctuations in blood pressure.

The rise in blood pressure which accompanies gross distension of the bladder could depend on a number of factors such as anxiety and other effects of pain and discomfort, distension of the hollow viscus, and contraction of sphincters. The results of our experiments suggest that the latter may be an important factor, at least when conscious effort is required to prevent micturition. It is possible that contraction of the sphincters initiates reflex activity affecting the cardiovascular system, but it is also probable that the primary volitional effort aimed at the sphincters should spill over into autonomic pathways leading to cardiovascular centres. This would be similar to the mechanism invoked by those people who can voluntarily accelerate heart rate, raise blood pressure, and stimulate other activities controlled by the autonomic nervous system (Ogden and Shock, 1939 ; Whyte, 1966).

\section{Summary}

Blood pressure rose significantly (average $27 / 20 \mathrm{~mm}$. $\mathrm{Hg}$ ) in seven normal subjects and four patients when the bladder was distended to the maximum tolerable limit by oral fluid loading or retrograde filling (four patients) through a catheter. Pulse rate was unaffected.

There was a positive correlation between blood pressure and mounting bladder pressure for the combined results from the four catheterized patients, but this relation did not obtain in each individual.

There was no significant difference in blood pressures taken immediately before and after micturition when subjects followed their habitual practice in determining when to micturate.

Blood pressure rose significantly (average $17 / 12 \mathrm{~mm} . \mathrm{Hg}$ ) whenever subjects without distended bladders voluntarily contracted their sphincters. Pulse rate also rose.

\section{REFERENCES}

Adams-Ray, J., and Norlén, G. (1951). Acta physiol. scand., 23, 95. Cunningham, D. J. C., Guttmann, L., Whitteridge, D., and Wyndham. C. H. (1953). F. Physiol. (Lond.), 121, 581.

Guttmann, L and Whitteridge, D. (1947). Brain, 70, 361.

Lyle, C. B., Monroe, J. T., Flinn, D. E., and Lamb, L. E. (1961). Ner Engl. f. Med., 265, 982.

O'Conor, V. J. (1920). Arch. Surg., 1, 359.

Ogden, E., and Shock, N. W. (1939). Amer. f. med. Sci., 198, 329.

Ogden, E., and Shock, N. W. (1939). Amer. 7.

Watkins, A. L. (1938). Amer. F. Physiol., 121, 32

Whitteridge, D. (1960). Physiol. Rev., 40, Suppl. No. 4, p. 198

Whyte, H. M. (1966). Unpublished.

\title{
Multiple Sclerosis and Vaccination
}

\author{
HENRY MILLER,* M.D., F.R.C.P. ; WOJCIECH CENDROWSKI,† M.D. ; KURT SCHAPIRA,† M.D
}

Brit. med. F., 1967, 2, 210-213

The purpose of this paper is to describe nine examples of onset or exacerbation of multiple sclerosis after vaccination or inoculation and to discuss the possible implications of these observations. The first five cases were seen in Newcastle and the remainder in Pruszkow. In five instances the onset of the neurological disease occurred after inoculation, in four (Cases $3,4,5$, and 9) this was followed by exacerbation of established multiple sclerosis. The cases are summarized in the Table.

\begin{tabular}{|c|c|c|c|c|c|c|}
\hline $\begin{array}{l}\text { Case } \\
\text { No. }\end{array}$ & $\begin{array}{l}\text { Age at } \\
\text { Onset }\end{array}$ & Sex & $\begin{array}{c}\text { Vaccinated } \\
\text { Against }\end{array}$ & $\begin{array}{l}\text { Period of } \\
\text { Latency }\end{array}$ & \multicolumn{2}{|c|}{$\begin{array}{l}\text { Subsequent } \\
\text { Clinical Course }\end{array}$} \\
\hline \multirow{6}{*}{$\begin{array}{l}1 \\
2 \\
3^{*} \\
4^{*} \\
5^{*} \\
6 \\
7 \\
8 \\
9^{*}\end{array}$} & \multirow{6}{*}{$\begin{array}{l}23 \\
22 \\
19 \\
26 \\
21 \\
41 \\
13 \\
31 \\
30\end{array}$} & \multirow{6}{*}{$\begin{array}{l}\mathbf{F} \\
\mathbf{M} \\
\mathbf{M} \\
\mathbf{F} \\
\mathbf{F} \\
\mathbf{F} \\
\mathbf{F} \\
\mathbf{F} \\
\mathbf{F}\end{array}$} & \multirow{2}{*}{$\begin{array}{l}\text { Smallpox } \\
\text { Yellow fever } \\
\text { Typhoid fever }\end{array}$} & 48 hours & \multicolumn{2}{|c|}{ Remittent, mild } \\
\hline & & & & $\begin{aligned} & \text { Few } \\
& 12\end{aligned}$ & " & " \\
\hline & & & Smälnox " & 14 days & , & moderate \\
\hline & & & \multirow{2}{*}{$\begin{array}{l}\text { Smallpox } \\
\text { Rabies } \\
\text { Tuberculosis } \\
\text { Typhoid fever }\end{array}$} & 48 hours & ". & moderate \\
\hline & & & & $\begin{array}{c}7-14 \text { days } \\
14 \text { ” }\end{array}$ & $\ddot{.}$ & \\
\hline & & & 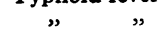 & Few & $\therefore$ & erat: \\
\hline
\end{tabular}

\section{Case Reports}

\section{Case 1}

In 1945 , five years after primary vaccination, a previously healthy 23-year-old woman was revaccinated against smallpox. Within 48 hours she developed dragging of the left leg, clumsiness of the left hand, and dysuria, all of which cleared up within a few months. One year later, in 1946, she developed a left retrobulbar neuritis, in 1949 the right eye was similarly affected, and in 1953 there was a recurrence affecting the left. Examination in 1957 revealed bilateral optic atrophy, peripheral blunting of sensation in the lower limbs, absent abdominal reflexes, and a right extensor plantar response. Disability was slight.

\section{Case 2}

In 1942 a 22 -year-old man, who had received primary vaccination against smallpox, T.A.B., and tetanus toxoid inoculation six weeks previously without ill effect, was inoculated against yellow fever. This was followed within hours by progressive failure of vision in both eyes, more severe in the left, which recovered within a few weeks. A year later he had a mild attack of retrobulbar neuritis on the right. Between 1944 and 1951 he had about a dozen recurrent episodes of numbness on either side of the body, hemiparesis, and emotionalism. Examination in 1957 revealed left optic atrophy, left intention tremor, absence of the abdominal responses, slight ataxia of gait, and euphoria, with little disablement.

\section{Case 3}

In 1955 , at the age of 45,26 years after a right facial palsy and 18 years after a transient diplopia, a man was secondarily vaccinated

\footnotetext{
* Department of Neurology, Royal Victoria Infirmary, Newcastle upon

Tyne, England.
+ Neurological Clinic of the Psychoneurological Institute, Pruszkow. Warsaw, Poland.

$\ddagger$ Department of Psychological Medicine, Royal Victoria Infirmary, Newcastle upon Tyne, England.
} 